\title{
Effect of $\mathrm{NaCl}$ additive on properties of aqueous PEG-sodium sulfate two-phase system
}

\author{
Luisa A. Ferreira ${ }^{\mathrm{a}, \mathrm{c}, 1}$, Pier Parpot ${ }^{\mathrm{b}, 2}$, José A. Teixeira ${ }^{\mathrm{a}, 3}$, Larissa M. Mikheeva ${ }^{\mathrm{c}, 4}$, Boris Y. Zaslavsky ${ }^{\mathrm{c}, *}$ \\ a IBB - Institute for Biotechnology and Bioengineering, Centre of Biological Engineering, Universidade do Minho, Campus de Gualtar, 4710-057 Braga, Portugal \\ b Departamento de Química, Universidade do Minho, Campus de Gualtar, 4710-057 Braga, Portugal \\ ${ }^{c}$ Analiza, Inc., 3615 Superior Ave., Cleveland, $\mathrm{OH} 44114$, USA
}

\section{A R T I C L E I N F O}

\section{Article history:}

Received 28 October 2011

Received in revised form

29 November 2011

Accepted 1 December 2011

Available online 8 December 2011

\section{Keywords:}

Aqueous two-phase systems

Salt additives

Non-ionic solutes partitioning

Solvatochromic solvent features

Solute dipolarity/polarizability

Solute hydrogen bond acidity

Solute hydrogen bond basicity

\begin{abstract}
A B S T R A C T
The concentrations of all components in the phases of aqueous two-phase polyethylene glycol-sodium sulfate system of a fixed composition with different concentrations of $\mathrm{NaCl}$ additive were determined. Solvatochromic solvent features of aqueous media in the phases of all the systems were characterized in terms of solvent dipolarity/polarizability, solvent hydrogen bond donor acidity and hydrogen bond acceptor basicity. Partitioning of a homologous series of dinitrophenylated amino acids with aliphatic alkyl side chain was examined in all the systems, and the differences between the relative hydrophobicity and electrostatic properties of the phases were quantified. These differences were described in terms of solvatochromic solvent features of the phases. The previously reported partition coefficients of twelve different nonionic compounds in all the systems were expressed in terms of solute descriptors. It is demonstrated that two solvatochromic solvent descriptors (solvent dipolarity/polarizability, and solvent hydrogen bond donor acidity) could adequately describe the partitioning of the solutes in all the systems employed.
\end{abstract}

(c) 2011 Elsevier B.V. All rights reserved.

\section{Introduction}

Aqueous two-phase systems (ATPS) are formed by the mixing of two (or more) water-soluble polymers or a single polymer and specific salt in an aqueous solution above certain critical concentrations or temperature. As a result, two immiscible aqueous phases are formed. These systems are unique because each of the phases is over $80 \%$ water on a molal basis and yet the phases are immiscible and differ in their solvent properties [1-4]. Therefore they can be used for the differential distribution of added solutes. ATPS have been used for the separation of biological macromolecules and particles for over 50 years [5-7].

ATPS formed by polyethylene glycol (PEG) and inorganic salt, such as sodium sulfate or phosphate, are commonly used for protein purification due to their low cost and good operational characteristics [5,6]. Extraction in ATPS has been demonstrated as

\footnotetext{
* Corresponding author. Tel.: +1 216432 9050x111; fax: +1 2164329050. E-mail addresses: laferreira@deb.uminho.pt (L.A. Ferreira), parpot@quimica.uminho.pt (P. Parpot), jateixeira@deb.uminho.pt (J.A. Teixeira), lar@analiza.com (L.M. Mikheeva), bz@analiza.com (B.Y. Zaslavsky).

1 Tel.: +351 253604420; fax: +351253604429.

2 Tel.: +351 253604057; fax: +351 253678983

3 Tel.: +351 253604406; fax: +351253604429.

4 Tel.: +1 216432 9050x106; fax: +1 2164329050 .
}

an efficient method for large scale recovery and purification of biological products [7-10]. Low cost, high capacity and easy scale-up are clear advantages of this technology. For successful utilization of partitioning in ATPS it is important to understand the mechanisms of solute distribution in the systems as well as system properties at the molecular level.

It is well established that the solute partitioning in two-polymer and polymer-salt ATPS is affected by similar factors. Salt additives are among the factors which are known to strongly influence the solute partitioning in two-polymer ATPS [1]. It is also known that salt additives may affect protein partitioning in polymer-salt ATPS [8-10]. The most widely used salt additive in these ATPS is $\mathrm{NaCl}$ generally employed in the concentration range up to $15 \% \mathrm{wt}$., i.e. up to ca. $2.6 \mathrm{M}[8-10]$.

Recently we reported on the effect of $\mathrm{NaCl}$ in the concentration range of $0-1.9 \mathrm{M}$ on phase diagram and partition behavior of a variety of nonionic compounds in PEG-8000-sodium sulfate ATPS [11]. The differences in binodial position observed for the ATPS with different $\mathrm{NaCl}$ concentrations failed to explain the effect of $\mathrm{NaCl}$ on partition behavior of nonionic organic compounds. In this study we examined concentrations of all components of ATPS in the coexisting phases, evaluated the differences between the relative hydrophobicity and electrostatic properties of the phases, and estimated solvatochromic solvent features of the aqueous media in the phases. We also evaluated solute descriptors for the organic 
compounds examined previously within the framework of the linear free energy relationship (LFER) model of solute-solvent interactions.

\section{Experimental}

\subsection{Materials}

Polyethylene glycol-8000 (Lot 048K00241) with an average molecular weight $\left(\mathrm{M}_{\mathrm{W}}\right)$ of 8000 was purchased from Sigma-Aldrich (St. Louis, MO, USA). The solvatochromic probes 4-nitrophenol (reagent grade, $>98 \%$ ) and 4-nitroanisole $(>97 \%, \mathrm{GC})$ were both purchased from Aldrich (Milwaukee, WI, USA) Reichardt's carboxylated betaine dye, 2,6-diphenyl-4-[2,6-diphenyl-4-(4carboxyphenyl)-1-pyridino]phenolate, sodium salt was kindly provided by Professor C. Reichardt (Philipps University, Marburg, Germany).

All salts and other chemicals used were of analytical-reagent grade.

\subsection{Methods}

\subsubsection{Phase diagrams of aqueous two-phase systems}

Stock solutions of PEG 8000 (50wt.\%) and $\mathrm{Na}_{2} \mathrm{SO}_{4}$ (20.3 wt.\%) were prepared in water. Sodium phosphate buffer ( $\mathrm{NaPB} ; 0.5 \mathrm{M}$, pH 6.8) was prepared by mixing $3.45 \mathrm{~g}$ of $\mathrm{NaH}_{2} \mathrm{PO}_{4} \cdot \mathrm{H}_{2} \mathrm{O}$ and $3.55 \mathrm{~g}$ $\mathrm{Na}_{2} \mathrm{HPO}_{4}$ in $100 \mathrm{~mL}$ aqueous solution. Stock solutions of $\mathrm{NaCl}$ of 0.5 and $5.0 \mathrm{M}$ concentration were prepared.

Each aqueous two-phase system of total weight of $25 \mathrm{~g}$ was prepared by weight in $50 \mathrm{~mL}$ graduated centrifuge tube. All ATPS had a fixed composition of 11.10 wt.\% PEG-8000, 6.33 wt.\% $\mathrm{Na}_{2} \mathrm{SO}_{4}$ and $0.01 \mathrm{M} \mathrm{NaPB}, \mathrm{pH} 6.8$, with different concentrations of $\mathrm{NaCl}$ additive from $0.027 \mathrm{M}$ up to $1.9 \mathrm{M}$, as previously described [11]. All weighing was carried out on an analytical balance precise to within $\pm 0.2 \mathrm{mg}$ (OHAUS, Explorer Pro, model EP214DC, Switzerland).

After vigorous mixing the systems were centrifuged for $30 \mathrm{~min}$ at $3000 \times g$ in a centrifuge (HERMLE, Z36HK, Germany) to speed the phase settling, and placed in a thermostatic bath $\left(23^{\circ} \mathrm{C}\right)$ for at least $24 \mathrm{~h}$ to equilibrate. Aliquots from both phases were withdrawn and used for characterization and for solvatochromic analysis (next section).

The concentrations of sodium sulfate, phosphate and chloride were determined by ion-exchange chromatography (Dionex DX100 , with a column Ion Pac AS11-HC Analytical ( $4 \mathrm{~mm} \times 250 \mathrm{~mm})$ ). Eluent solution was prepared by dissolving appropriate amounts of sodium hydroxide ( $\mathrm{NaOH}$, Panreac) in water. Calibration for each salt was performed at five concentrations evenly distributed in the range of $0.01-0.05 \mathrm{mM}$ for $\mathrm{Na}_{2} \mathrm{SO}_{4}, 0.02-0.2 \mathrm{mM}$ for $\mathrm{NaPB}$, and $0.002-0.02 \mathrm{mM}$ for $\mathrm{NaCl}$. Deionized water from Millipore (Milli-Q) was used for the preparation of all solutions. The precision of the IC method was assessed in terms of repeatability and intermediate (inter-day) precision by analyzing three replicate determinations on the same day and determinations on two different days with the same sample of salt solution. The calibration curve for each salt showed a linear relationship between the peak area and salt concentration over the aforementioned salt concentration range with a correlation coefficient $r^{2}>0.999$.

All anions were separated completely with total analysis time of $30 \mathrm{~min}$. Anions in the sample were identified by the coincidence of the corresponding retention times with those established for calibration and the peak areas were used for quantitative analysis.

Polymer amount in each phase was determined gravimetrically as an average from dry weights of three lyophilized water-diluted aliquots from each phase. The polymer concentration in each phase was calculated by subtraction of the total salt concentration in the phase from the average dry weight.

\subsubsection{Solvatochromic studies}

Solvatochromic parameters are used to conveniently describe solvent properties using additive or other models. Such parameters may include polarity/polarizability $\pi^{*}$, H-bond acceptor basicity $\beta$, and H-bond donor acidity $\alpha$.

The ATPS of the compositions listed in Table 1 were prepared as described above. The phases were separated and used for solvatochromic analysis. The solvatochromic probes 4-nitroanisole, 4-nitrophenol and Reichardt's carboxylated betaine were used to measure the solvent polarity/polarizability $\pi^{*}$, H-bond acceptor basicity $\beta$, and $\mathrm{H}$-bond donor acidity $\alpha$ in both phases of each particular ATPS. Three to four concentrations of each probe were prepared for each analysis in order to check for aggregation effects and specific interactions with the phase forming polymer and salt. $\mathrm{NaOH}(1 \mathrm{M})$ was added to the samples $(\sim 20 \mu \mathrm{L} \mathrm{NaOH} 1 \mathrm{M}$ to $1.1 \mathrm{~mL}$ of the phase) containing the Reichardt's carboxylated betaine to ensure a basic $\mathrm{pH}$. The samples were mixed thoroughly in a vortex mixer and then scanned in a Sinergy HT UV-Vis microplate spectrophotometer (Bio-Tek Instruments, Winooski, VT, USA) with a bandwidth of $2.0 \mathrm{~nm}$; data interval $1 \mathrm{~nm}$; and high resolution scan $(\sim 0.5 \mathrm{~nm} / \mathrm{s})$. Pure phases containing no dye (blank) were scanned first to establish a baseline. The maximum wavelength was the average between all these scans. Average standard deviations for each measured wavelength were $\leq 0.7 \mathrm{~nm}$ for all probes. A few drops of $1 \mathrm{M} \mathrm{HCl}(\sim 20 \mu \mathrm{L} 1 \mathrm{M} \mathrm{HCl}$ to $1.1 \mathrm{~mL}$ phase) were added to the samples containing 4-nitrophenol in order to eliminate charge transfer bands that were observed in some systems.

The behavior of the probes (4-nitrophenol, and Reichardt's carboxylated betaine dye) in several solvents (water, n-hexane, methanol and some phases of ATPS) was tested in the presence and absence of $\mathrm{HCl}$ (for 4-nitrophenol) and $\mathrm{NaOH}$ (for the betaine dye) at different concentrations of the probes, acid or base, and the maximum shifts of the probes were within the experimental errors not exceeding $0.7 \mathrm{~nm}$ in all cases (data not shown) [4].

a) Determination of the solvent dipolarity/polarizability $\pi^{*}$

$\pi^{*}$ was determined from the wave number $\left(v_{(1)}\right)$ of the longest wavelength absorption band of the 4-nitroanisole dye using the relationship given by Marcus [12]:

$$
\pi^{*}=0.427\left(34.12-v_{(1)}\right)
$$

b) Determination of the solvent hydrogen-bond acceptor basicity $\beta$

$\beta$ values were determined from the wave number $\left(v_{(2)}\right)$ of the longest wavelength absorption band of the 4-nitrophenol dye using the relationship [12]:

$$
\beta=0.346\left(35.045-v_{(2)}\right)-0.547 \pi^{*}
$$

c) Determination of the solvent hydrogen-bond donor acidity $\alpha$ $\alpha$ values were determined from the longest wavelength absorption band of Reichardt's betaine dye using the relationship [12]:

$$
\alpha=0.0649 E_{\mathrm{T}}(30)-2.03-0.72 \pi^{*}
$$

The $E_{\mathrm{T}}(30)$ values are based on the negatively solvatochromic pyridinium $\mathrm{N}$-phenolate betaine dye as probe molecule, and are obtained directly from the wavelength absorption band of the carboxylated form as

$E_{\mathrm{T}}(30)=\frac{1}{0.932}\left(\frac{28591}{\lambda-3.335}\right)$

where $\lambda$ is the wavelength in $\mathrm{nm}$. 
Table 1

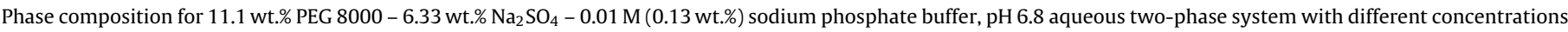
of $\mathrm{NaCl}$ additive (all concentrations in wt.\% except when indicated).

\begin{tabular}{|c|c|c|c|c|c|c|c|c|c|c|c|}
\hline \multicolumn{2}{|l|}{ Feed } & \multicolumn{4}{|c|}{ Upper phase } & \multicolumn{4}{|c|}{ Lower phase } & \multirow[t]{2}{*}{$\mathrm{TLL}^{\mathrm{a}}$} & \multirow[t]{2}{*}{$\mathrm{STL}^{\mathrm{a}}$} \\
\hline $\mathrm{NaCl}, \mathrm{M}$ & $\mathrm{NaCl}$ & PEG & $\mathrm{Na}_{2} \mathrm{SO}_{4}$ & $\mathrm{NaPB}^{\mathrm{a}}$ & $\mathrm{NaCl}$ & PEG & $\mathrm{Na}_{2} \mathrm{SO}_{4}$ & $\mathrm{NaPB}^{\mathrm{a}}$ & $\mathrm{NaCl}$ & & \\
\hline 0 & 0 & 19.50 & 3.47 & 0.081 & 0 & 0.80 & 9.74 & 0.213 & 0 & 19.72 & -2.98 \\
\hline 0.215 & 1.15 & 23.75 & 2.20 & 0.059 & 1.16 & 0.682 & 9.68 & 0.200 & 1.23 & 24.25 & -3.09 \\
\hline 0.543 & 2.89 & 24.68 & 1.94 & 0.053 & 2.46 & 0.660 & 9.63 & 0.200 & 3.11 & 25.22 & -3.12 \\
\hline 1.109 & 5.83 & 26.79 & 1.35 & 0.050 & 5.24 & 0.576 & 9.82 & 0.192 & 6.11 & 27.55 & -3.10 \\
\hline 1.936 & 9.91 & 30.29 & 1.04 & 0.033 & 7.08 & 0.613 & 9.37 & 0.204 & 10.80 & 30.83 & -3.56 \\
\hline
\end{tabular}

a $\mathrm{NaPB}$ - sodium phosphate buffer, pH 6.8; TLL - tie-line length defined as TLL $=\left(\Delta[\mathrm{PEG}]^{2}+\Delta\left[\mathrm{Na}_{2} \mathrm{SO}_{4}\right]^{2}\right)^{0.5}$ where $\Delta$ denotes the difference between the concentrations of the indicated components in the coexisting phases; STL-tie-line slope defined as STL $=\Delta[\mathrm{PEG}] / \Delta\left[\mathrm{Na}_{2} \mathrm{SO}_{4}\right]$.

\subsubsection{Partitioning}

A semi-automated methodology for performing aqueous twophase partitioning, was used to measure the partition coefficients of sodium salts of dinitrophenylated (DNP) amino acids with aliphatic side-chains (glycine, alanine, norvaline, norleucine, and $\alpha$-aminon-caprylic acid) in all the ATPS. DNP-amino acids solutions of $10 \mathrm{mM}$ were prepared in $10 \mathrm{mM} \mathrm{NaOH}$ and the exposure to light minimized. Varied amounts (e.g. 0, 20, 40, 60, 80, and $100 \mu \mathrm{L}$ ) of a given compound solution and the corresponding amounts (e.g. $100,80,60,40,20$, and $0 \mu \mathrm{L}$ ) of water were added using a Multipette Xstream pipette (Eppendorf, Hamburg, Germany) to a set of the same polymer/buffer/salt mixtures, with a final system weight of ca. $0.5 \mathrm{~g}$. Systems were vortexed and centrifuged for $30 \mathrm{~min}$ at $3000 \times g$ in a centrifuge (HERMLE, Z36HK, Germany) to accelerate phase settling. Aliquots of $20-70 \mu \mathrm{L}$ from the upper and lower phases were withdrawn and diluted with appropriate amount of water. Following vortexing, $300 \mu \mathrm{L}$ aliquots were transferred into microplate wells and a synergy HT UV-Vis microplate reader (Bio-Tek Instruments) was used to measure optical absorbance at $362 \mathrm{~nm}$. Phases of blank systems at corresponding dilutions were measured for comparison.

The partition coefficient, $K$, is defined as the ratio of the sample concentration in the upper phase to the sample concentration in the lower phase. The $K$-value for each solute was determined as the slope of the concentration in the upper phase plotted as a function of the concentration in the bottom phase averaged over the results obtained from six partition experiments carried out at different concentrations of the solute and at the fixed composition of the system taking into account different dilutions used for the phases. Deviation from the average $K$-value was consistently below $5 \%$ and in most cases lower than $2 \%$.

\section{Results and discussion}

\subsection{Composition of the phases}

Concentrations of all components of each ATPS in the upper and lower phases are listed in Table 1. It follows from the data presented in Table 1 that increasing the $\mathrm{NaCl}$ additive concentration is followed by an increase of the tie-line length for the system of the fixed PEG and $\mathrm{Na}_{2} \mathrm{SO}_{4}$ overall composition though the tie-line slope (definition of both see as a footnote in Table 1 ) does not change up to $1.109 \mathrm{M} \mathrm{NaCl}$ concentration. Analysis of the data in Table 1 indicates that addition of various amounts of $\mathrm{NaCl}$ to the $\mathrm{PEG}-\mathrm{Na}_{2} \mathrm{SO}_{4}$ ATPS of an otherwise fixed composition results in redistribution of all the components between the coexisting phases. The interrelationship between the components may be described as:

$$
\begin{gathered}
\Delta[\mathrm{PEG}]=0.2_{ \pm 2.3}-1.2_{ \pm 0.18} \Delta[\mathrm{NaCl}]+3.0_{ \pm 0.31} \Delta\left[\mathrm{Na}_{2} \mathrm{SO}_{4}\right] \\
N=5 ; R^{2}=0.9943 ; \mathrm{SD}=0.43 ; F=174.8
\end{gathered}
$$

where $\Delta[\mathrm{PEG}], \Delta\left[\mathrm{Na}_{2} \mathrm{SO}_{4}\right]$ and $\Delta[\mathrm{NaCl}]$ differences between the concentrations of the corresponding components in the upper and lower phases; $N$ is the number of ATPS examined; $R^{2}$ is the correlation coefficient; $F$ is the ratio of variance; $S D$ is the standard deviation.

It should be mentioned that Eq. (5) describes empirical relationship between the differences in the concentrations of the components in the two phases of the particular ATPS examined. The interrelationship described by Eq. (5) indicates that addition $\mathrm{NaCl}$ to the PEG- $\mathrm{Na}_{2} \mathrm{SO}_{4}$ ATPS of the fixed composition results in the changes of all the concentrations of all the components in the coexisting phases.

\subsection{Difference between the relative hydrophobicity and electrostatic properties of the phases}

In order to characterize the solvent properties of the coexisting phases of the ATPS with varied concentrations of $\mathrm{NaCl}$ additive we evaluated the differences between the relative hydrophobicity and electrostatic properties of the phases. For this purpose we measured the partition coefficients of sodium salts of dinitrophenylated (DNP) amino acids with aliphatic side-chains (alanine, norvaline, norleucine, and $\alpha$-amino-n-caprylic acid) in all ATPS. Typical experimental data obtained for sodium salts of DNP-amino acids in different ATPS are plotted in Fig. 1, and the linear curves observed may be described as:

$\ln K_{\mathrm{DNP}-\mathrm{AA}}^{(i)}=C^{(i)}+E^{(i)} N_{\mathrm{C}}$

where $K_{\text {DNP-AA }}$ is the partition coefficient of a DNP-amino acid with aliphatic side-chain; superscript $(i)$ denotes the particular $i$-th ATPS used for the partition experiments; $N_{\mathrm{C}}$ is equivalent number of $\mathrm{CH}_{2}$ groups in the aliphatic side-chain of a given DNP-amino acid; $E$ is

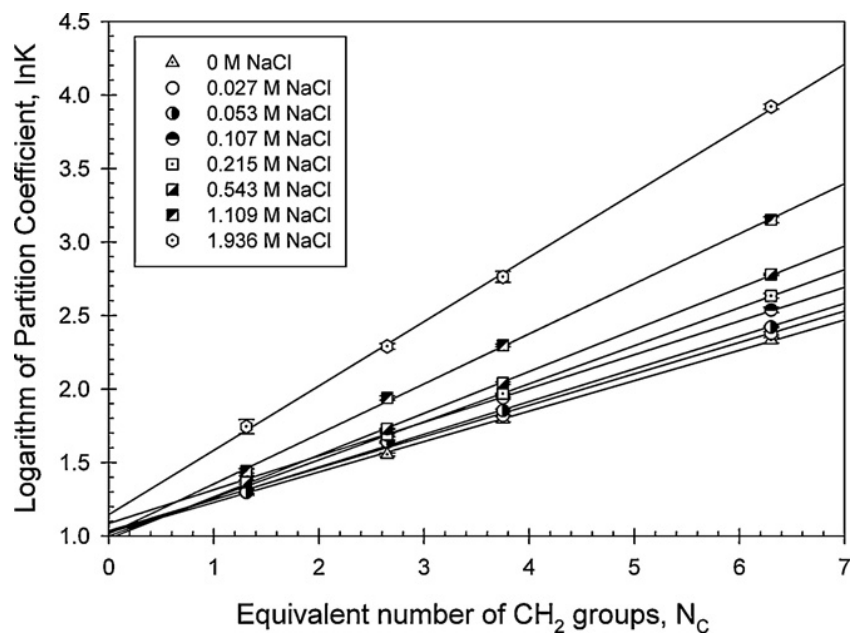

Fig. 1. Partition coefficients of sodium salts of DNP-amino acids with aliphatic sidechains as functions of side-chain lengths in aqueous PEG-8000- $\mathrm{Na}_{2} \mathrm{SO}_{4}$ two-phase system with indicated concentration of $\mathrm{NaCl}$ additive ( $\mathrm{pH} 6.8$; temperature $23^{\circ} \mathrm{C}$ ). 
Table 2

Coefficients E and C (see Eq. (6) in text) and the free energy of transfer of a $\mathrm{CH}_{2}$ group between the coexisting phases of PEG-Na $\mathrm{SO}_{4}$ ATPS of a fixed composition with indicated concentration of $\mathrm{NaCl}$ additive.

\begin{tabular}{llll}
\hline $\mathrm{NaCl}, \mathrm{M}$ & $E$ & $C$ & $-\Delta G\left(\mathrm{CH}_{2}\right), \mathrm{cal} / \mathrm{mole}$ \\
\hline 0 & $0.207 \pm 0.003$ & $1.024 \pm 0.012$ & $122 \pm 1.8$ \\
0.027 & $0.214 \pm 0.006$ & $1.035 \pm 0.023$ & $126 \pm 3.5$ \\
0.053 & $0.223 \pm 0.005$ & $1.024 \pm 0.017$ & $131 \pm 2.9$ \\
0.107 & $0.230 \pm 0.002$ & $1.086 \pm 0.008$ & $135 \pm 1.2$ \\
0.215 & $0.259 \pm 0.001$ & $1.002 \pm 0.005$ & $152 \pm 0.6$ \\
0.543 & $0.284 \pm 0.002$ & $0.983 \pm 0.008$ & $167 \pm 1.2$ \\
1.109 & $0.341 \pm 0.004$ & $1.015 \pm 0.016$ & $200 \pm 2.4$ \\
1.936 & $0.438 \pm 0.006$ & $1.146 \pm 0.022$ & $258 \pm 3.5$
\end{tabular}

an average $\ln \mathrm{K}$ increment per $\mathrm{CH}_{2}$ group; $C$ represents the total contribution of the non-alkyl part of the structure of a DNP-amino acid into $\ln K_{\text {DNP-AA }}$.

The coefficients $E^{(i)}$ and $C^{(i)}$ values determined for the ATPS examined are listed in Table 2. It should be noted here that the partition coefficient of a DNP-amino acid, $K_{\text {DNP-AA }}$, was determined in each ATPS as the ratio of the solute concentration in the top phase to that in the bottom phase. As the standard free energy of transfer of a solute between the coexisting phases is described as:

$\Delta G^{0}=-R T \ln K$

where $R$ is the universal gas constant and $T$ is the absolute temperature in Kelvin, it follows that

$\Delta G^{0}\left(\mathrm{CH}_{2}\right)=-R T E$

where $\Delta G^{0}\left(\mathrm{CH}_{2}\right)$ is the standard free energy of transfer of a methylene group from one phase to another. The $\Delta G^{0}\left(\mathrm{CH}_{2}\right)$ values calculated from the experimental data with Eqs. (6)-(8) are listed in Table 2.

Examination of the parameter $E$ or $\Delta G^{0}\left(\mathrm{CH}_{2}\right)$ values in Table 2 indicates that the difference between the relative hydrophobicity of the phases increases with increasing concentration of $\mathrm{NaCl}$ additive. Since changes in the $\mathrm{NaCl}$ concentration affects the composition of both phases in the system, it is necessary to explore the solvent features of the media in the phases to gain better insight into this effect.

\subsection{Solvatochromic solvent descriptors and models}

Each of the solvent parameters $\pi^{*}, \beta$, and $\alpha$ were obtained from a set of single solvatochromic probes as reported previously [4]. The solvatochromic parameters measured in each phase of the ATPS and the differences between their values in the upper and lower phases are presented in Table 3. First analysis of solvatochromic features of the aqueous media in PEG-2000-salt ATPS (salt-ammonium sulfate and potassium phosphate) was reported by Huddleston et al. [13]. It was found [13] that the differences between the solvent $\mathrm{H}$-bond acceptor basicity $(\Delta \beta)$ and solvent dipolarity/polarizability $\left(\Delta \pi^{*}\right)$ of the phases were both small, and only the difference between the solvent $\mathrm{H}$-bond donor acidity $(\Delta \alpha)$ was readily measurable. The authors [13] concluded that in PEG-salt ATPS the principal molecular determinants of partition behavior of a solute are such solute properties as size, $\mathrm{H}$-bond acceptor basicity, and aromaticity or halogenicity. Further analysis of partitioning of multiple solutes in PEG-2000- $\left(\mathrm{NH}_{4}\right)_{2} \mathrm{SO}_{4}$ ATPS [2] was performed within the framework of the Abraham model of solute-solvent interactions [14-17] using the following description of the solute partition coefficient:

$\log K=z_{0}+r R_{2}+s S+b A+a B+v V_{x}$

where the solute descriptors are: $V_{x}$ is the solute McGowan volume; $R_{2}$ is the solute excess molar refraction; $S$ is the solute dipolarity/polarizability, and $A$ and $B$ are the solute hydrogen bond donor acidity and hydrogen bond acceptor basicity, respectively; $z_{0}$ is constant, and $r, s, b, a$, and $v$ represent the relative solvent properties of the phases corresponding to the appropriate solute descriptors.

The approach employed by Huddleston et al. [2] is to examine partition coefficients of compounds with known solute descriptors and calculate the solvent characteristics for a given two-phase system as unknown constants in Eq. (10) by multiple linear regression analysis. The following LSER relationship was reported by Huddleston et al. for PEG-2000-( $\left.\mathrm{NH}_{4}\right)_{2} \mathrm{SO}_{4}$ ATPS [2]:

$$
\begin{aligned}
\log K= & -0.05+0.65 R_{2}-0.21 S-1.31 B+0.21 A \\
& +1.71 V x \quad N=29 ; R^{2}=0.97 ; F=187.58
\end{aligned}
$$

where the solute descriptors are as defined above.

It was shown [2], however, that the above relationship may be simplified as:

$\log K=0.5 R_{2}-1.22 B+1.70 V_{x} \quad N=29 ; R^{2}=0.97 ; F=265.31$.

In this study we used the approach opposite to the one employed by Huddleston et al. [2] and previously employed for analysis of solute partitioning in different two-polymer ATPS of the same ionic composition [4]. This approach is based on using solvent characteristics measured as solvatochromic solvent features, and the solute descriptors being calculated as unknown constants for a given solute with partition coefficients measured in different ATPS. The partition coefficient of a nonionic solute in this case is successfully described as:

$\log K=z_{0}+S \Delta \pi^{*}+A \Delta \beta+B \Delta \alpha$

where $S, A$, and $B$ are the solute descriptors, $z_{0}$ is the constant, and $\Delta \pi^{*}, \Delta \beta$, and $\Delta \alpha$ are experimentally determined solvent features defined above.

The parameter $E$ (Table 2) related to the standard free energy of transfer of a $\mathrm{CH}_{2}$ group between the phases generally considered $[1-4,18,19]$ to be equivalent to the difference between the free energy of cavity formation in the phases is described in terms of solvent solvatochromic features as:

$$
\begin{gathered}
E=0.101_{ \pm 0.008}-1.8_{ \pm 0.45} \Delta \pi^{*}-2.5_{ \pm 0.88} \Delta \beta-0.73_{ \pm 0.040} \Delta \alpha, \\
N=6 ; R^{2}=0.994 ; \mathrm{SD}=0.004 ; F=113.07
\end{gathered}
$$

(number of ATPS is limited to $N=6$ due to the lack of $\Delta \alpha$ values for ATPS with highest $\mathrm{NaCl}$ additive concentrations). It must be stressed that parameter $\mathrm{E}$ was calculated in natural logarithm units (see Eq. (6)). It also seems reasonable that the free energy of cavity formation in an aqueous medium, resulting in rearrangement of the highly cooperative hydrogen-bonds network, would involve all types of solvent-solvent interactions.

All of the ATPS under study differ significantly in the concentrations of salts in the coexisting phases (see Table 1). It was shown [19] that in order to describe partitioning of ionized solutes in two-polymer ATPS with a fixed ionic composition an additional parameter capable of quantifying the difference between the electrostatic properties of the phases has to be included in Eq. (12). It was suggested by Zaslavsky [1, pp. 208-216] to use the contribution of an ionic group into the solute partition coefficient as an empirical measure of the difference in question. The experimental results here were obtained with sodium salts of p-dinitrophenyl-amino acids, i.e. compounds possessing a DNP-NH-CH $\left(\mathrm{C}_{n} \mathrm{H}_{2 n+1}\right)-\mathrm{COO}^{-} \mathrm{Na}^{+}$group. This moiety is bulky and contains a substituted aromatic ring. Use of this particular group as a probe for electrostatic ion-ion, ion-dipole and dipole-dipole interactions obviously has some limitations. Only to a first approximation the standard free energy of transfer of this group between 
Table 3

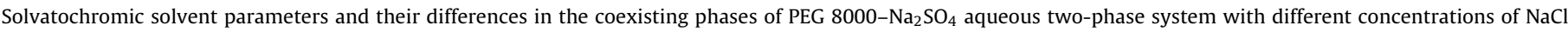
additive and a fixed composition of 11.10 wt.\% PEG-8000, 6.33 wt.\% $\mathrm{Na}_{2} \mathrm{SO}_{4}$ and $0.01 \mathrm{M}(0.13 \mathrm{wt} . \%)$ sodium phosphate buffer, pH 6.8

\begin{tabular}{|c|c|c|c|c|c|c|c|c|c|}
\hline \multirow[t]{2}{*}{$\mathrm{NaCl}, \mathrm{M}$} & \multicolumn{3}{|c|}{ Upper phase } & \multicolumn{3}{|c|}{ Lower phase } & \multirow[t]{2}{*}{$\Delta \pi^{*}$} & \multirow[t]{2}{*}{$\Delta \beta$} & \multirow[t]{2}{*}{$\Delta \alpha$} \\
\hline & $\pi^{*}$ & $\beta$ & $\alpha$ & $\pi^{*}$ & $\beta$ & $\alpha$ & & & \\
\hline 0 & 1.113 & 0.661 & 0.924 & 1.142 & 0.646 & 1.052 & -0.029 & 0.015 & -0.128 \\
\hline 0.027 & 1.121 & 0.649 & 0.930 & 1.147 & 0.640 & 1.051 & -0.026 & 0.009 & -0.121 \\
\hline 0.053 & 1.114 & 0.650 & 0.928 & 1.122 & 0.650 & 1.075 & -0.008 & 0.000 & -0.147 \\
\hline 0.107 & 1.097 & 0.665 & 0.940 & 1.182 & 0.626 & 1.042 & -0.085 & 0.039 & -0.102 \\
\hline 0.215 & 1.152 & 0.648 & 0.889 & 1.179 & 0.635 & 1.078 & -0.027 & 0.013 & -0.189 \\
\hline 0.543 & 1.155 & 0.643 & 0.880 & 1.183 & 0.631 & 1.106 & -0.028 & 0.012 & -0.226 \\
\hline 1.109 & 1.142 & 0.659 & 0.865 & 1.188 & 0.635 & a & -0.046 & 0.024 & $\mathrm{a}$ \\
\hline 1.936 & 1.126 & 0.687 & 0.845 & 1.144 & 0.668 & a & -0.018 & 0.019 & $\mathrm{a}$ \\
\hline
\end{tabular}

a The diffuse absorption spectrum for Reichardt's carboxylated betaine observed in the salt-rich phase precluded determination of the maximum wavelength.

the coexisting phases of an ATPS may be viewed as a measure of the ability of aqueous media to participate in a particular kind of intermolecular interactions. This parameter is represented by parameter $C$ in Eq.(6), and was included in the modified linear solvation energy relationship Eq. (12) used successfully for description of proteins partitioning in ATPS [19].

In the PEG- $\mathrm{Na}_{2} \mathrm{SO}_{4}$ ATPS studied here parameter $C$ may be described as:

$$
\begin{gathered}
C=1.09_{ \pm 0.019}-0.6_{ \pm 0.18} \Delta \pi^{*}+0.5_{ \pm 0.10} \Delta \alpha, \\
N=6 ; R^{2}=0.956 ; \mathrm{SD}=0.009 ; F=32.4 .
\end{gathered}
$$

It must be stressed here that parameter $C$ was calculated in natural logarithm units (Eq. (6)). It should also be noted that the difference between the solvent hydrogen bond acceptor basicity is not included in Eq. (14).

We used Eq. (12) to determine the solute descriptors from the partition coefficients of nonionic compounds in the PEG$8000-\mathrm{Na}_{2} \mathrm{SO}_{4}$ ATPS with different concentrations of $\mathrm{NaCl}$ additive reported previously [11]. The solute descriptors values obtained are presented in Table 4. It may be seen from the values of the solute descriptors that the solute dipolarity/polarizability and the solute $\mathrm{H}$-bond donor acidity are linearly interrelated as:

$$
\begin{aligned}
& A_{i}^{\text {eq } 12}=0.4_{ \pm 0.13}+1.50_{ \pm 0.025} * S_{i}{ }^{\text {eq } 12} \\
& \quad N=12 ; R^{2}=0.9967 ; \mathrm{SD}=0.23 ; F=3702.4
\end{aligned}
$$

where $A_{i}^{\text {eq } 12}$ and $S_{i}^{\text {eq } 12}$ are the $i$-th solute H-bond donor acidity and dipolarity/polarizability, respectively (both calculated with Eq. (12)); $\mathrm{N}$ is the number of solutes examined; other parameters as defined above.
Relationship described by Eq. (15) implies that the partition coefficient of a nonionic solute in the ATPS examined may be described by the simplified equation as:

$\log K=z_{0}+S \Delta \pi^{*}+B \Delta \alpha$

where all parameters are as defined above.

It should be noted that the relative solvent $\mathrm{H}$-bond donor acidity of the aqueous media in the phases appears to be one of the two most important factors governing the nonionic solute partitioning in PEG-sodium sulfate ATPS, in agreement with the data reported by Huddleston et al. [2].

We used Eq. (16) to determine the solute descriptors from the partition coefficients of nonionic compounds in the PEG$8000-\mathrm{Na}_{2} \mathrm{SO}_{4}$ ATPS with different concentrations of $\mathrm{NaCl}$ additive reported previously [11]. The solute descriptors values obtained are presented in Table 5.

Comparison of the solute descriptors values listed in Tables 4 and 5 shows that the solute descriptor $B$ values are essentially independent of what equation - Eqs. (12) or (16) is used for description of the solute partition coefficient. The solute descriptor $S$ values, however, differ significantly when calculated with Eqs. (12) or (16) likely due to the interrelationship described by Eq. (15). It should also be indicated that the descriptors values for same solutes (glucoside, mannoside, caffeine, phenol, 3-hydroxy-benzaldehyde, and 4-hydroxy-acetanilide) determined in a variety of ATPS formed by different pairs of polymers at the same ionic composition of $0.15 \mathrm{M} \mathrm{NaCl}$ in $0.01 \mathrm{M}$ sodium phosphate buffer, pH 7.4 [4] are significantly different from those determined in this work. The likely reason for these differences may be that the polar nonionic compounds partitioned in the PEG- $\mathrm{Na}_{2} \mathrm{SO}_{4}$ ATPS interact with the aqueous media at rather high concentrations of salts $(\sim 0.8 \mathrm{M}$ to $\sim 3.0 \mathrm{M}$ in the lower phase and

\begin{tabular}{|c|c|c|c|c|c|c|c|c|}
\hline Solute & $z_{0}^{\mathrm{eq} 12}$ & $S^{e q 12}$ & $A^{\text {eq12 }}$ & $B^{\text {eq12 }}$ & $N$ & $F$ & $\mathrm{SE}$ & $R^{2}$ \\
\hline Glucoside $^{\mathrm{b}}$ & $0.186 \pm 0.009$ & $-2.2 \pm 0.51$ & $-3 \pm 1$ & $-0.98 \pm 0.046$ & 6 & 159.7 & 0.004 & 0.996 \\
\hline Caffeine & $0.156 \pm 0.014$ & $-1.5 \pm 0.75$ & $-2 \pm 1.5$ & $-0.76 \pm 0.068$ & 6 & 43.8 & 0.006 & 0.985 \\
\hline 3-Hydroxybenzaldehyde & $0.345 \pm 0.010$ & $-6.3 \pm 0.56$ & $-9 \pm 1.1$ & $-2.16 \pm 0.05$ & 6 & 631.8 & 0.004 & 0.999 \\
\hline Phenol & $0.316 \pm 0.009$ & $-7.4 \pm 0.47$ & $-10.9 \pm 0.93$ & $-2.32 \pm 0.043$ & 6 & 1000.6 & 0.004 & 0.999 \\
\hline Mannoside ${ }^{c}$ & $0.182 \pm 0.008$ & $-2.4 \pm 0.43$ & $-3.2 \pm 0.85$ & $-1.04 \pm 0.039$ & 6 & 246.8 & 0.003 & 0.997 \\
\hline Trihydroxybenzene & $0.499 \pm 0.023$ & $-10 \pm 1.2$ & $-14 \pm 2.4$ & $-3.1 \pm 0.11$ & 6 & 269.5 & 0.010 & 0.998 \\
\hline Adenosine & $0.250 \pm 0.009$ & $-3 \pm 0.47$ & $-4.1 \pm 0.92$ & $-1.15 \pm 0.042$ & 6 & 254.9 & 0.004 & 0.997 \\
\hline Salicin & $0.114 \pm 0.017$ & $-2.2 \pm 0.92$ & $-3 \pm 1.8$ & $-1.08 \pm 0.083$ & 6 & 59.5 & 0.007 & 0.989 \\
\hline 4-Nitroanisole & $0.331 \pm 0.024$ & $-7 \pm 1.3$ & $-11 \pm 2.6$ & $-2.3 \pm 0.12$ & 6 & 133.7 & 0.010 & 0.995 \\
\hline Guanosine & $0.111 \pm 0.023$ & $-2 \pm 1.2$ & $-2 \pm 2.4$ & $-0.7 \pm 0.11$ & 6 & 15.4 & 0.010 & 0.958 \\
\hline 4-Aminophenol & $0.270 \pm 0.012$ & $-4.2 \pm 0.65$ & $-6 \pm 1.3$ & $-1.40 \pm 0.058$ & 6 & 199.1 & 0.005 & 0.997 \\
\hline 4-Hydroxyacetanilide & $0.352 \pm 0.007$ & $-7.2 \pm 0.38$ & $-10.1 \pm 0.76$ & $-2.52 \pm 0.034$ & 6 & 1819.7 & 0.003 & 0.999 \\
\hline
\end{tabular}
$\sim 0.3 \mathrm{M}$ to $\sim 1.3 \mathrm{M}$ in the upper phase), i.e. in the concentration

Table 4

Solute descriptors for the indicated nonionic compounds calculated as regression coefficients in Eq. (12). ${ }^{\mathrm{a}}$

a Eq. (12) $\log K=z_{0}+S \Delta \pi^{*}+A \Delta \beta+B \Delta \alpha$ for indicated compounds.

b 4-Nitrophenyl- $\alpha$-D-glucopyranoside.

c 4-Nitrophenyl- $\alpha$-D-mannopyranoside. 
Table 5

Solute descriptors for the indicated nonionic compounds calculated as regression coefficients in Eq. (16). ${ }^{\mathrm{a}}$

\begin{tabular}{|c|c|c|c|c|c|c|c|}
\hline Solute & $z_{0}^{\text {eq } 16}$ & $S^{e q 16}$ & $B^{\text {eq16 }}$ & $N$ & $F$ & SE & $R^{2}$ \\
\hline Glucoside $^{b}$ & $0.20 \pm 0.014$ & $-0.9 \pm 0.13$ & $-0.95 \pm 0.074$ & 6 & 83.1 & 0.007 & 0.982 \\
\hline Caffeine & $0.16 \pm 0.013$ & $-0.6 \pm 0.12$ & $-0.74 \pm 0.068$ & 6 & 58.6 & 0.006 & 0.975 \\
\hline 3-Hydroxybenzaldehyde & $0.38 \pm 0.044$ & $-1.8 \pm 0.41$ & $-2 \pm 0.23$ & 6 & 39.5 & 0.021 & 0.963 \\
\hline Phenol & $0.36 \pm 0.052$ & $-1.9 \pm 0.49$ & $-2.2 \pm 0.28$ & 6 & 31.3 & 0.026 & 0.954 \\
\hline Mannoside ${ }^{c}$ & $0.20 \pm 0.017$ & $-0.8 \pm 0.16$ & $-1.0 \pm 0.087$ & 6 & 66.1 & 0.008 & 0.978 \\
\hline Trihydroxybenzene & $0.56 \pm 0.067$ & $-2.7 \pm 0.63$ & $-2.9 \pm 0.35$ & 6 & 34.6 & 0.033 & 0.959 \\
\hline Adenosine & $0.27 \pm 0.021$ & $-0.9 \pm 0.19$ & $-1.1 \pm 0.11$ & 6 & 51.3 & 0.010 & 0.972 \\
\hline Salicin & $0.13 \pm 0.017$ & $-0.9 \pm 0.16$ & $-1.05 \pm 0.09$ & 6 & 67.6 & 0.008 & 0.978 \\
\hline 4-Nitroanisole & $0.38 \pm 0.054$ & $-1.9 \pm 0.51$ & $-2.2 \pm 0.29$ & 6 & 29.1 & 0.027 & 0.951 \\
\hline Guanosine & $0.12 \pm 0.020$ & $-0.5 \pm 0.19$ & $-0.7 \pm 0.11$ & 6 & 23.1 & 0.010 & 0.939 \\
\hline 4-Aminophenol & $0.30 \pm 0.030$ & $-1.3 \pm 0.28$ & $-1.3 \pm 0.16$ & 6 & 36.3 & 0.015 & 0.960 \\
\hline 4-Hydroxyacetanilide & $0.40 \pm 0.049$ & $-2.1 \pm 0.46$ & $-2.4 \pm 0.26$ & 6 & 43.4 & 0.024 & 0.967 \\
\hline
\end{tabular}

a Eq. (16): $\log K=z_{0}+\mathrm{S} \Delta \pi^{*}+\mathrm{B} \Delta \alpha$ for indicated compounds.

b 4-Nitrophenyl- $\alpha$-D-glucopyranoside.

c 4-Nitrophenyl- $\alpha$-D-mannopyranoside.

range where salting-out effects of $\mathrm{NaCl}$ and $\mathrm{Na}_{2} \mathrm{SO}_{4}$ are commonly observed. It seems reasonable to suggest that specific ion-dipole interactions occurring at these salt concentrations would affect the solute descriptors values. The fact that the solute descriptors listed in Tables 4 and 5 for each compound are constant within all the PEG- $\mathrm{Na}_{2} \mathrm{SO}_{4}-\mathrm{NaCl}$ ATPS compositions used implies that these specific interactions are independent of the salts concentrations within the range employed. It should also be mentioned that all the solute descriptors under consideration, solute dipolarity/polarizability, and solute hydrogen bond acidity and hydrogen bond basicity are known to account partially for electrostatic solute-solvent interactions [20-22] and hence it seems reasonable that the values of these descriptors might change at high salt concentrations in solution.

Finally, it remains to be explored further if PEG-salt ATPS provide solute descriptors values useful for physicochemical analysis of solute-solvent interactions but these descriptors are clearly useful for predicting the solute partition behavior in the PEG-salt ATPS as shown in Fig. 2.

The partition coefficient values calculated from data in Table 4 according to Eq. (12) are in the perfect agreement with the experimental values reported previously [11]:

$$
\begin{aligned}
& \log K_{\text {calc }}=-0.002_{ \pm 0.0027}+1.002_{ \pm 0.004} K_{\text {exp }} \\
& N=72 ; R^{2}=0.9987 ; \mathrm{SD}=0.0095 ; F=55896.9
\end{aligned}
$$

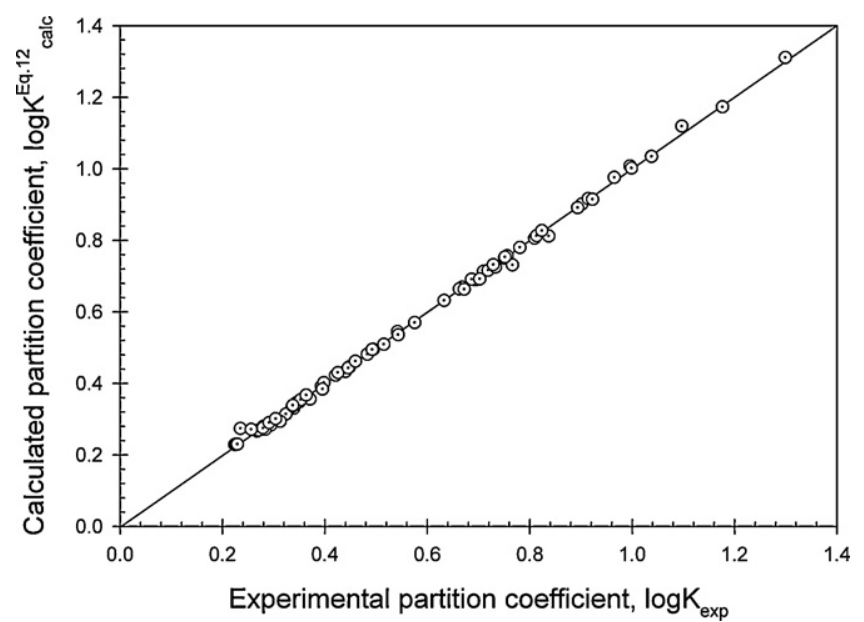

Fig. 2. Partition coefficients $K$, experimentally measured for all the examined compounds [11] in each ATPS (Table 1), plotted against $K$ values calculated with Eq. (12) using experimentally determined solvatochromic solvent parameters (Table 3 ) and solute descriptors presented in Table 4 ( $\mathrm{pH}$ 6.8; temperature $23^{\circ} \mathrm{C}$ ). where $K_{\exp }$ and $K_{\text {calc }}$ are the experimental partition coefficient value [11] and calculated partition coefficient value, respectively.

In the case of the partition coefficient values calculated from data in Table 5 according to simplified Eq. (16) there is also a good agreement with the experimental values [11]:

$$
\begin{aligned}
& \log K_{\text {calc }}=-0.002_{ \pm 0.004}+1.001_{ \pm 0.006} K_{\text {exp }} \\
& N=72 ; R^{2}=0.9972 ; \mathrm{SD}=0.014 ; F=24913
\end{aligned}
$$

where $K_{\exp }$ and $K_{\text {calc }}$ are the experimental partition coefficient value [11] and calculated partition coefficient value, respectively.

The results obtained in this study indicate unambiguously that solvatochromic solvent features of the phases enable prediction of partitioning of a nonionic solute in the ATPS, and allow one to compare ATPS of different composition in regard to their partition ability. We are currently working on characterization of solvent features of the aqueous media in the coexisting phases of the ATPS with other salt additives in order to gain better insight into the mechanism of solute partitioning in PEG-salt ATPS.

\section{Acknowledgment}

Luisa A. Ferreira acknowledges the financial support (SFRH/BPD/47607/2008) from Fundação para a Ciência e a Tecnologia (Lisboa, Portugal).

\section{References}

[1] B.Y. Zaslavsky, Aqueous Two-Phase Partitioning: Physical Chemistry and Bioanalytical Applications, Marcel Dekker, New York, 1994.

[2] H.D. Willauer, J.G. Huddleston, R.D. Rogers, Ind. Eng. Chem. Res. 41 (2002) 2591.

[3] M.L. Moody, H.D. Willauer, S.T. Griffin, J.G. Huddleston, R.D. Rogers, Ind. Eng. Chem. Res. 44 (2005) 3749.

[4] P.P. Madeira, C.A. Reis, A.E. Rodrigues, L.M. Mikheeva, B.Y. Zaslavsky, J. Phys. Chem. B 114 (2010) 457.

[5] P.A. Albertsson, Partition of Cell Particles and Macromolecules, 3rd ed., Wiley, New York, 1986

[6] H. Walter, D.E. Brooks, D. Fisher (Eds.), Partitioning in Aqueous Two-Phase Systems: Theory, Methods, Use, and Applications to Biotechnology, Academic Press, Orlando, FL, 1985.

[7] R. Hatti-Kaul (Ed.), Aqueous Two-Phase Systems. Methods and Protocols, Humana Press, Totowa, 2000.

[8] B.A. Andrews, S. Nielsen, J.A. Asenjo, Bioseparation 6 (1996) 303

[9] P.A.J. Rosa, A.M. Azevedo, S. Sommerfeld, M. Mutter, M.R. Aires-Barros, W. Backer, J. Biotechnol. 139 (2009) 306

[10] S.A. Oelmeier, F. Dismer, J. Hubbuch, Biotechnol. Bioeng. 108 (2011) 69.

[11] L.A. Ferreira, J.A. Teixeira, L.M. Mikheeva, A. Chait, B.Y.Zaslavsky, J. Chromatogr. A 1218 (2011) 5031.

[12] Y. Marcus, Chem. Soc. Rev. 22 (1993) 409

[13] J.G. Huddleston, H.D. Willauer, R.D. Rogers, Phys. Chem. Chem. Phys. 4 (2002) 4065.

[14] M.H. Abraham, H.S. Chadha, G.S. Whiting, R.C. Mitchell, J. Pharm. Sci. 83 (1994) 1085

[15] M.H. Abraham, Chem. Soc. Rev. 22 (1993) 73. 
[16] J.A. Platts, D. Butina, M.H. Abraham, A. Hersey, J. Chem. Inf. Comput. Sci. 39 (1999) 835.

[17] M.H. Abraham, J. Gil-Lostes, M. Fatemi, Eur. J. Med. Chem. 14 (2009) 2452.

[18] P. Madeira, J.A. Teixeira, E.A. Macedo, L.M. Mikheeva, B.Y. Zaslavsky, J. Chromatogr. A 1185 (2008) 85.
[19] P. Madeira, C.A. Reis, A.E. Rodrigues, L.M. Mikheeva, A. Chait, B.Y. Zaslavsky, J. Chromatogr. A 1218 (2011) 1379.

[20] O. Lamarche, J.A. Platts, A. Hersey, Phys. Chem. Chem. Phys. 3 (2001) 2747.

[21] O. Lamarche, J.A. Platts, Chem. Phys. Lett. 367 (2003) 123.

[22] O. Lamarche, J.A. Platts, A. Hersey, J. Chem. Inf. Comput. Sci. 44 (2004) 848. 